\title{
THE AUTOCORRELATION MATCHING METHOD FOR DISTRIBUTED MIMO COMMUNICATIONS OVER UNKNOWN FIR CHANNELS
}

\author{
Hui Luo ${ }^{1}$, Ruey-Wen Liu ${ }^{2}$, Xieting $\operatorname{Lin}^{3}, X i n L i^{3}$ \\ ${ }^{1}$ AT\&T Labs - Research, Red Bank, NJ 07701, USA \\ ${ }^{2}$ University of Notre Dame, Notre Dame, IN 46556, USA \\ ${ }^{3}$ Fudan University, Shanghai, China
}

\begin{abstract}
The Autocorrelation Matching method is a blind signal separation and channel equalization technique for distributed MIMO communication systems over unknown FIR channels using only second order statistics. This method is based on a theoretical discovery, i.e., under the condition that the autocorrelation functions of the (multiple) inputs are linearly shift-independent, an input is recovered, up to a unitary factor and a delay, by an output of an MIMOFIR equalizer if and only if the autocorrelation function of the output matches that of the input. An optimal zero-forcing equalizer is developed to maximize the SNR for the outputs, i.e., the recovered inputs. Some preliminary simulation results show that the BER in the recovered inputs is about $3 \times 10^{-5}$ at the $\mathrm{SNR}=15$ $\mathrm{dB}$. This method has the potential to be applied to cellular wireless communications for the purpose of boosting spectrum efficiency or suppressing co-channel interference.
\end{abstract}

\section{INTRODUCTION}

Recently, MIMO (Multiple Input Multiple Output) communications techniques have been under intensive study, in light of the ultra high spectrum efficiency that MIMO communications systems can achieve. The essence of MIMO communications is: (1) using multiple transmitters to transmit multiple signals over the same carrier simultaneously as if each signal would have occupied the channel exclusively; and (2) using some signal processing technique to separate the individual transmitted signals from the received mixtures out of a receiving antenna array. Theoretically, the channel capacity (bits per second per $\mathrm{Hz}$ ) for an MIMO communications system is roughly proportional to the number of the transmitters [2]. A pioneer prototype developed by Bell Labs scientists has demonstrated such ultra high spectrum efficiency in lab environment [3].

Up to now, the research of MIMO communications has been dominated by that of space-time coding techniques, which has made tremendous success in new communications theory development [10]. The principle of space-time coding techniques is to introduce coding redundancy in the multiple transmitted signals in both space dimension (in terms of cross-correlations between the multiple transmitted signals) and time dimension (in terms of autocorrelations in the individual transmitted signals). This redundancy information will then help to recover the transmitted symbols after the transmitted signals are separated from the MIMO channel that is identified using training sequences. Clearly, in order to apply space-time coding techniques, all transmitters in an MIMO communications system must be synchronized.
As a supplement for space-time coding techniques, a new signal processing scheme, called the Autocorrelation Matching (AM) method, is proposed in this paper for distributed MIMO communications over unknown FIR channels, where it is difficult or impossible to synchronize distributed transmitters. At each transmitter, the AM method introduces distinctive information in the autocorrelations of the transmitted signal. At the receiver, the individual transmitted signals can be recovered from the received mixtures by matching the autocorrelations between the processed signals and the transmitted signals.

The AM method is a blind signal separation and channel equalization method. That is, it doesn't rely on training sequences to identify the MIMO-FIR channel. Instead, it bypasses the process of channel identification and directly achieves the signal separation and channel equalization by adjusting the parameters of an MIMO-FIR equalizer at the receiver until the autocorrelations of the output signals of this equalizer matches those of the transmitted signals.

Furthermore, the AM method is a blind signal separation and channel equalization method suitable for wireless communications. Differing from many celebrated blind signal separation and channel equalization methods based on high-order statistics (HOS) [5, $6,11]$, the AM method only needs to estimate a set of shifted second order statistics (SOS) of the received signals. This is important, because wireless channels are fast time-varying and thus often only a couple hundreds of signal samples are available before the channel characteristics have changed significantly, which are sufficient for SOS estimation but insufficient for HOS estimation. In addition, compared with other elegant blind signal separation and channel equalization methods based on cyclo-stationary SOS $[1,12]$ or SOS $[7,13]$, the AM method needs less conditions on transmitted signals and MIMO-FIR channels.

The AM method has the potential to be applied to cellular wireless communications in two scenarios. (1) If a base station has multiple antennas but mobile stations have only single antennas, the uplink can form a distributed MIMO communications system and thus multiple mobile stations can transmit signals to the base station over the same carrier. In this case, the AM method can significantly improve the uplink spectrum efficiency. (2) If a mobile station has multiple antennas, the downlink from desired base station and that from interfering base stations can also form a distributed MIMO communications model. In this case, the AM method can be used to suppress co-channel interference.

The rest of this paper presents the system model, the theoretic fundamental, and an optimal algorithm for the AM method. 


\section{SYSTEM MODEL}

A distributed MIMO communications system based on the AM method is given in Fig. 1 .

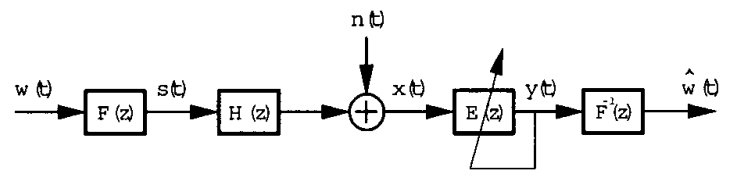

Fig.1 A distributed MIMO communications system

where,

$$
\begin{array}{ll}
\mathbf{w}(t): & \begin{array}{l}
\text { an } N \times 1 \text { white source signal. } \\
\mathbf{w}(t)=\left[w_{1}(t), w_{2}(t), \cdots, w_{N}(t)\right]^{T}
\end{array} \\
\mathbf{F}(z): \quad \begin{array}{l}
\text { an } N \times N \text { diagonal pre-filter to be designed. } \\
\mathbf{F}(z)=\operatorname{diag}\left(f_{1}(z), f_{2}(z), \cdots, f_{N}(z)\right)
\end{array} \\
\mathbf{s}(t): \quad \begin{array}{l}
\text { an } N \times 1 \text { transmitted signal. } \\
\mathbf{s}(t)=\left[s_{1}(t), s_{2}(t), \cdots, s_{N}(t)\right]^{T}
\end{array} \\
\mathbf{H}(z): \quad \begin{array}{l}
\text { an }(\text { unknown } M \times N \text { FIR channel. } \\
\mathbf{H}(z)=\left[\sum_{l=0}^{L_{h}} h_{m n}^{(l)} z^{-l}\right]_{m, n=1,1}^{M, N}=\sum_{l=0}^{L_{h}} \mathbf{H}_{l} z^{-l}
\end{array} \\
\mathbf{n}(t): \quad \begin{array}{l}
\text { an }(\text { unknown } M \times 1 \text { additive noise. } \\
\mathbf{n}(t)=\left[n_{1}(t), n_{2}(t), \cdots, n_{M}(t)\right]^{T}
\end{array} \\
\mathbf{x}(t): \quad \begin{array}{l}
\text { an } M \times 1 \text { received signal. } \\
\mathbf{x}(t)=\left[x_{1}(t), x_{2}(t), \cdots, x_{M}(t)\right]^{T}
\end{array} \\
\mathbf{E}(z): \quad \begin{array}{l}
\text { an } N \times M \text { FIR equalizer to be designed. } \\
\mathbf{E}(z)=\left[\sum_{l=0}^{L_{e}} e_{n m}^{(l)} z^{-l}\right]_{n, m}^{N, M}
\end{array} \\
\mathbf{y}(t): \quad \begin{array}{l}
\text { an } N \times 1 \text { processed signal. } \\
\text { a }(t)=\left[y_{1}(t), y_{2}(t), \cdots, y_{N}(t)\right]^{T}
\end{array}
\end{array}
$$

The system can be described by,

$$
\left\{\begin{array}{l}
\mathbf{s}(t)=\mathbf{F}(z)[w(t)] \\
\mathbf{x}(t)=\mathbf{H}(z)[s(t)]+\mathbf{n}(t) \\
\mathbf{y}(t)=\mathbf{E}(z)[\mathbf{x}(t)]=\mathbf{C}(z)[\mathbf{s}(t)]+\mathbf{E}(z)[\mathbf{n}(t)]
\end{array}\right.
$$

where, $t \in Z$. The polynomial matrices, $\mathbf{F}(z), \mathbf{H}(z), \mathbf{E}(z)$, and $\mathbf{C}(z)$, act as convolution operators in these equations, e.g.,

$$
\mathbf{H}(z)[\mathbf{s}(t)]=\sum_{l=0}^{L_{h}} \mathbf{H}_{l} \mathbf{s}(t-l)
$$

The pre-filter $\mathbf{F}(z)$ has a diagonal form, which means no crosscorrelations between transmitted signals. The composite system response, $\mathbf{E}(z) \mathbf{H}(z)$, is represented by an $N \times N$ polynomial matrix $\mathbf{C}(z)$ with a degree of $L=L_{h}+L_{e}$.

The objective of the AM mothod is to design a pre-filter $\mathbf{F}(z)$ and a blind equalizer $\mathbf{E}(z)$ that matches the autocorrelations of the transmitted signal $\mathbf{s}(t)$ with those of the processed signal $y(t)$, such that the following zero-forcing condition is satisfied,

$$
\mathbf{C}(z)=\operatorname{diag}\left(d_{1} z^{-l_{1}}, d_{2} z^{-l_{2}}, \ldots, d_{N} z^{-l_{N}}\right)
$$

with $\left|d_{n}\right|=1$ and $0 \leq l_{n} \leq L$ for every $n=1,2, \ldots, N$. Note that the actual sequence of the transmitted signal $\mathbf{s}(t)$ and the MIMO-FIR channel $\mathbf{H}(z)$ (including $L_{h}$, the degree of $\mathbf{H}(z)$ ) are unknown in the above process.

The following assumptions are needed throughout this paper. They will not be repeated in theorem statements.

AS1 $w(t)$ is a wide-sense stationary vector sequence with zero mean and, without loss of generality, unit variance.
AS2 $\mathbf{w}(t)$ is spatially uncorrelated and, without loss of generality, temporally white, i.e., $E\left(\mathbf{w}(t) \mathbf{w}^{H}(t-k)\right)=\delta(k) \mathbf{I}$.

AS3 $|\mathbf{C}(z)| \neq 0$ for some $z$.

AS4 $\mathbf{n}(t)$ is a wide-sense stationary vector sequence with zero mean and unknown variance $\left[\sigma_{1}^{2}, \sigma_{2}^{2}, \cdots, \sigma_{M}^{2}\right]^{T}$.

AS5 $\mathbf{n}(t)$ is spatially uncorrelated and temporally white, i.e., $E\left(\mathbf{n}(t) \mathbf{n}^{H}(t-k)\right)=\delta(k) \operatorname{diag}\left(\sigma_{1}^{2}, \sigma_{2}^{2}, \cdots, \sigma_{M}^{2}\right)$.

\section{PRELIMINARIES}

First, the existence of an MIMO-FIR equalizer $\mathbf{E}(z)$ is not guaranteed for any MIMO-FIR channel $\mathbf{H}(z)$. A sufficient and necessary condition for existing an MIMO-FIR equalizer that can equalize an MIMO-FIR channel is given below [9], which is weaker than that $\mathbf{H}(z)$ is irreducible.

\section{Existence Condition}

The greatest common divisor (GCD) of all $N \times N$ determinants of $\mathbf{H}(z)$ is a monomial with regard to $z^{-1}$, i. e.,

$$
\operatorname{GCD}\left\{\left|\Delta \mathbf{H}_{i}(z)\right|, i=1,2, \ldots, C_{M}^{N}\right\}=z^{-l}
$$

for some $l \geq 0$. Where, $\Delta \mathbf{H}_{i}(z)$ 's are $N \times N$ submatrices of $\mathbf{H}(z) . C_{M}^{N}$ is the combination number of choosing $N$ out of $M$.

Secondly, the existence of an MIMO-FIR equalizer $\mathrm{E}(z)$ does not guarantee the existence of a such equalizer with any given degree. There exists a lower bound for the equalizer degree. However, it is not easy to find a small lower bound, because the lower bound depends on the characteristics of the MIMO-FIR channel $\mathbf{H}(z)$. Per practical reasons, we suggest an empirical lower bound $N L_{h} /(M-N)-1$. See [4] for further reference.

Thirdly, it has been found that an MIMO-FIR channel equalization is not always guaranteed when the SOS of the processed signals are the same as that of the transmitted signals. Nevertheless, this is guaranteed when the set of autocorrelation functions of transmitted signals, $\left\{r_{s_{n}}(\tau)=E\left(s_{n}(t) s_{n}^{*}(t-\tau)\right) \mid n=\right.$ $1,2, \cdots, N\}$, is linearly shift-independent with length $L[8]$, i.e.,

\section{Linear Shift-Independence Condition}

The set of shifted autocorrelation functions of the transmitted signals, $\left\{r_{s_{n}}(\tau-l) \mid n=1,2, \cdots, N ; l=0, \pm 1, \cdots, \pm L\right\}$, is linearly independent.

The following theorem is the fundamental of the AM method.

\section{Theorem 1}

Assume $\mathbf{n}(t)=\mathbf{0}$ and $\left\{r_{s_{n}}(\tau) \mid n=1,2, \cdots, N\right\}$ is linearly shift-independent with length $L$. Then $y_{1}(t)=d_{1} z^{-l_{1}}\left[s_{1}(t)\right]$ if and only if $y_{1}(t)$ has the same autocorrelations as does $s_{1}(t)$. (proof omitted)

\section{PRINCIPLE}

The AM method consists of two steps: (1) designing a diagonal pre-filter $\mathbf{F}(z)$ such that the autocorrelation functions of the transmitted signal $\mathbf{s}(t)$ satisfy the linear shift-independence condition; and (2) designing an MIMO-FIR equalizer $\mathbf{E}(z)$ such that the autocorrelations of the transmitted signal $\mathbf{s}(t)$ match those of the processed signal $\mathbf{y}(t)$.

The pre-filtering effects can be reversed after the transmitted signals are separated. 


\subsection{Pre-filters}

It is an art to design pre-filters. Two pre-filters are given below due to their simplicities in structure.

The two-tap FIR pre-filter is given by,

$$
\mathbf{F}(z)=\operatorname{diag}\left(1,1+b z^{-(L+1)}, \ldots, 1+b z^{-(N-1)(L+1)}\right)
$$

where $0<|b|<1$. This pre-filter is similar to the correlative filter introduced by [13]. It is considered the simplest FIR prefilter because each filter has only two taps (except the first one) and only one parameter is needed.

The homogeneous FIR pre-filter is given by

$$
\mathbf{F}(z)=\operatorname{diag}\left(f_{1}(z), f_{2}(z), \cdots, f_{N}(z)\right)
$$

in which each filter is defined as,

$$
f_{n}(z)=\prod_{m=1, N}^{m \neq n}\left(1-b_{m} z^{-(L+1)}\right)
$$

where, $b_{m} \in R, 0<\left|b_{m}\right|<1$ for $m=1,2, \cdots, N$, and $b_{1} \neq$ $b_{2} \neq \cdots \neq b_{N}$.

The simplest blind MIMO-FIR equalization problem is to separate two signals. In this special case, there is a general criterion of designing FIR pre-filters, as shown by the following theorem.

\section{Theorem 2}

The pre-filter $\mathbf{F}(z)=\operatorname{diag}\left(f_{1}(z), f_{2}(z)\right)$ makes the autocorrelation functions of $s_{1}(t)$ and $s_{2}(t)$ linearly shift-independent with length $L$, if $f_{1}(z)$ and $f_{2}(z)$ have no common zeros and if at least one of their degrees is greater than $L$. (proof omitted)

\subsection{Optimal Zero-Forcing Equalizer}

The noise issue needs to be addressed in the optimal zero-forcing equalizer design. Let an $M\left(L_{\dot{e}}+1\right) \times 1$ vector $\mathrm{e}_{1}$ stand for a zero-forcing equalizer for the transmitted signal $s_{1}(t)$. We have,

$$
y_{1}(t)=d_{1} s_{1}\left(t-l_{1}\right)+\overline{\mathbf{e}}_{1}^{T} \overline{\mathbf{n}}(t)
$$

where,

$$
\begin{gathered}
\overline{\mathbf{e}}_{1}=\left[\tilde{\mathbf{e}}_{11}^{T}, \tilde{\mathbf{e}}_{12}^{T}, \cdots, \tilde{\mathbf{e}}_{1 M}^{T}\right]^{T} \\
\overline{\mathbf{n}}(t)=\left[\overline{\mathbf{n}}_{1}^{T}(t), \tilde{\mathbf{n}}_{2}^{T}(t), \cdots, \tilde{\mathbf{n}}_{M}^{T}(t)\right]^{T}
\end{gathered}
$$

in which

$$
\begin{gathered}
\tilde{\mathbf{e}}_{1 m}=\left[e_{1 m}^{(0)}, e_{1 m}^{(1)}, \cdots, e_{1 m}^{\left(L_{e}\right)}\right]^{T} \\
\tilde{\mathbf{n}}_{m}(t)=\left[n_{m}(t), n_{m}(t-1), \cdots, n_{m}\left(t-L_{e}\right)\right]^{T}
\end{gathered}
$$

for $m=1,2, \cdots, M$. Calculating the autocorrelations on both side, we have,

$$
r_{y_{1}}(k)=r_{s_{1}}(k)+\overline{\mathbf{e}}_{1}^{T} \mathbf{D}(k) \overline{\mathbf{e}}_{1}^{*}
$$

where, $\mathbf{D}(k)$ is an $M\left(L_{e}+1\right) \times M\left(L_{e}+1\right)$ matrix,

$$
\mathbf{D}(k)=\operatorname{diag}\left(\sigma_{1}^{2} \mathbf{J}^{k}, \sigma_{2}^{2} \mathbf{J}^{k}, \cdots, \sigma_{M}^{2} \mathbf{J}^{k}\right)
$$

in which $\mathbf{J}$ is an $\left(L_{e}+1\right) \times\left(L_{e}+1\right)$ shifting matrix,

$$
\mathbf{J}=\left[\begin{array}{cccc}
0 & 0 & \cdots & 0 \\
1 & 0 & \ddots & \vdots \\
\vdots & \ddots & \ddots & 0 \\
0 & \cdots & 1 & 0
\end{array}\right]
$$

Note that $\mathbf{J}^{-k}$ stands for $\left(\mathbf{J}^{T}\right)^{k}$ for $k>0$ and $\mathbf{J}^{0}=\mathbf{I}$. Let $k=0$ in $\mathrm{Eq}(1)$, we have,

$$
r_{y_{1}}(0)=r_{s_{1}}(0)+\overline{\mathbf{e}}_{1}^{T} \mathbf{D}(0) \overline{\mathbf{e}}_{1}^{*}
$$

where, the left side is the power of the processed signal $y_{1}(t)$, a recovered version of the transmitted signal $s_{1}(t)$; the first term at the right side is the power of the transmitted signal $s_{1}(t)$; and the second term at the right side is the noise power contained in the recovered signal $y_{1}(t)$.

Clearly, the optimal zero-forcing blind equalizer is the one that minimizes the noise power contained in the recovered signal in addition to matching the autocorrelations between the recovered signal and the transmitted signal (after subtracting the difference caused by the noise power). That is, the optimal blind equalizer for recovering $s_{1}(t)$ is a solution to $\mathrm{e}_{1}$ that minimizes the following two objectives simultaneously,

$$
\begin{aligned}
& J_{1}\left(\overline{\mathbf{e}}_{1}\right)=\sum_{k=0}^{N(2 L+1) / 2}\left|\overline{\mathbf{e}}_{1}^{T} \mathbf{R}_{\overline{\mathbf{x}}}(k) \overline{\mathbf{e}}_{1}^{*}-r_{s_{1}}(k)-\overline{\mathbf{e}}_{1}^{T} \mathbf{D}(k) \overline{\mathbf{e}}_{1}^{*}\right|^{2} \\
& J_{2}\left(\overline{\mathbf{e}}_{1}\right)=\overline{\mathbf{e}}_{1}^{T} \mathbf{D}(0) \overline{\mathbf{e}}_{1}^{*}
\end{aligned}
$$

where, $\mathbf{R}_{\overline{\mathbf{x}}}(k)$ is the autocorrelation matrix regarding a vector signal $\overline{\mathbf{x}}(t)$,

$$
\mathbf{R}_{\overline{\mathbf{x}}}(k)=E\left(\overline{\mathbf{x}}(t) \overline{\mathbf{x}}^{H}(t-k)\right)
$$

and the vector signal $\overline{\mathbf{x}}(t)$ is arranged from the received signal $\mathbf{x}(t)$,

in which

$$
\overline{\mathbf{x}}(t)=\left[\tilde{\mathbf{x}}_{1}^{T}(t), \overline{\mathbf{x}}_{2}^{T}(t), \cdots, \tilde{\mathbf{x}}_{M}^{T}(t)\right]^{T}
$$

$$
\tilde{\mathbf{x}}_{m}(t)=\left[x_{m}(t), x_{m}(t-1), \cdots, x_{m}\left(t-L_{e}\right)\right]^{T}
$$

for $m=1,2, \cdots, M$.

Since the noise variance $\left[\sigma_{1}^{2}, \sigma_{2}^{2}, \cdots, \sigma_{M}^{2}\right]^{T}$ is unknown, we turn them into variables to be optimized,

$$
\begin{aligned}
\lambda_{0} & =\sqrt{\overline{\mathbf{e}}_{1}^{T} \mathbf{D}(0) \overline{\mathbf{e}}_{1}^{*}} \\
\lambda_{k} & =\overline{\mathbf{e}}_{1}^{T} \mathbf{D}(k) \overline{\mathbf{e}}_{1}^{*} \text { for } k=1,2, \cdots, L_{e}
\end{aligned}
$$

Thus, the minimization problem (2) is equivalent to,

$$
\begin{aligned}
& \text { Minimize } J\left(\overline{\mathbf{e}}_{1}, \lambda_{0}, \lambda_{1}, \cdots, \lambda_{L_{e}}\right) \\
& \text { Subject to } \lambda_{0}^{2}>\left|\lambda_{1}\right|,\left|\lambda_{2}\right|, \cdots,\left|\lambda_{L_{e}}\right|
\end{aligned}
$$

where,

$$
\begin{aligned}
& J\left(\overline{\mathbf{e}}_{1}, \lambda_{0}, \lambda_{1}, \cdots, \lambda_{L_{e}}\right)=\left(1+\lambda_{0}^{2}\right) \sum_{k=0}^{N(2 L+1) / 2} \\
& \left|\overline{\mathbf{e}}_{1}^{T} \mathbf{R}_{\overline{\mathbf{x}}}(k) \overline{\mathbf{e}}_{1}^{*}-r_{s_{1}}(k)-\lambda_{0}^{2} \delta(k)-\sum_{l=1}^{L_{e}} \lambda_{l} \delta(k-l)\right|^{2}
\end{aligned}
$$

In practice, we typically have,

$$
\lambda_{0}^{2}>>\left|\lambda_{1}\right|,\left|\lambda_{2}\right|, \cdots,\left|\lambda_{L_{e}}\right|
$$

Hence, the minimization problem (3) can be solved approximately by minimizing the following objective,

$$
J\left(\overline{\mathbf{e}}_{1}, \lambda_{0}\right)=\left(1+\lambda_{0}^{2}\right) \sum_{k=0}^{N(2 L+1) / 2}\left|\overline{\mathbf{e}}_{1}^{T} \mathbf{R}_{\overline{\mathbf{x}}}(k) \overline{\mathbf{e}}_{1}^{*}-r_{s_{1}}(k)-\lambda_{0}^{2} \delta(k)\right|^{2}
$$

The final minimization problem (4) can be easily solved using an iterative gradient algorithm. 


\section{COMPUTER SIMULATION}

A computer simulation is conducted to separate two transmitted signals from five received mixtures. For the purpose of simplicity, only real numbers are used.

In the simulation, two uncorrelated white random sequences on $[-1,1]$ are generated as two source signals. They are filtered by the following pre-filters respectively,

$$
\left\{\begin{array}{l}
f_{1}(z)=1-0.3 z^{4}+0.5 z^{5} \\
f_{2}(z)=1+0.8 z^{4}+0.2 z^{5}
\end{array}\right.
$$

and then transmitted through the following MIMO-FIR channel,

$$
\mathbf{H}(z)=\left[\begin{array}{cc}
(1-0.89 z)(1+0.20 z) & (1+0.18 z)(1+0.76 z) \\
(1+0.12 z)(1-0.79 z) & (1+0.60 z)(1+0.72 z) \\
(1-0.84 z)(1-0.82 z) & (1-0.32 z)(1+0.50 z) \\
(1+0.86 z)(1+0.81 z) & (1-0.74 z)(1-0.83 z) \\
(1-0.38 z)(1-0.08 z) & (1+0.27 z)(1-0.57 z)
\end{array}\right]
$$

The received mixtures are equalized by the following MIMO-FIR equalizer,

$$
\mathbf{E}(z)=\mathbf{E}_{0}+\mathbf{E}_{1} z
$$

which is solved by an iterative gradient algorithm using the SOS of the received signals that are estimated from about 100 received symbols. After the transmitted signals are separated, they are reversely filtered and then converted into hard symbols 1 or -1 .

The BER vs. SNR curve is shown below, which is obtained from $2 \times 10^{5}$ test runs.

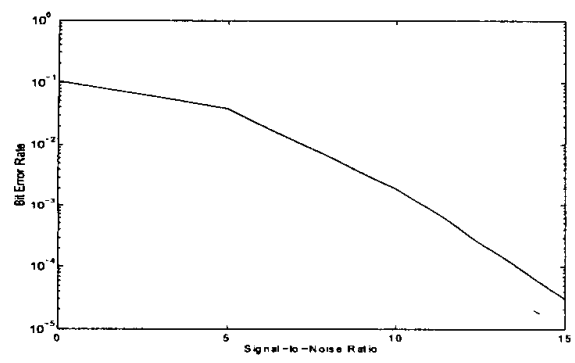

Fig.2 BER performance for the AM method

\section{CONCLUSIONS}

The Autocorrelation Matching method is presented in this paper, which is an SOS-based blind signal separation and channel equalization technique for distributed MIMO communication systems. It consists of two steps: (1) design a pre-filter such that each transmitted signal has a distinct autocorrelation function and the autocorrelation functions of all transmitted signals are linearly shiftindependent; and (2) design an MIMO-FIR equalizer that makes the autocorrelation functions of the processed signals match those of the transmitted signals. The AM method is based on a theoretic discovery, i.e., for transmitted signals with linearly shiftindependent autocorrelation functions, a processed signal is equal to a transmitted signal up to a unitary factor and a delay if and only if they have the same autocorrelation functions. An optimal zero-forcing equalizer is developed to maximize the SNR for the recovered transmitted signals. Some preliminary simulation results show that the BER in the recovered signal is about $3 \times 10^{-5}$ at the $\mathrm{SNR}=15 \mathrm{~dB}$. This method has the potential to be applied to cellular wireless communications for the purpose of boosting spectrum efficiency or suppressing co-channel interference.

\section{REFERENCES}

[1] A. Chevreuil and P. Loubaton, "MIMO Blind Second-Order Equalization Method and Conjugate Cyclostationary", IEEE Trans. on Signal Processing, vol. 47, no. 2, pp. 572-578, 1999.

[2] G. Foschini and M. Gans, "On Limits of Wireless Communications in a Fading Environment When Using Multiple Antennas", Wireless Personal Communications Magazine, vol. 6, no. 3, pp. 331-335, 1998.

[3] G. Foschini, "Layered Space-Time Architecture for Wireless Communication in a Fading Environment When Using Multiple Antennas", Bell Labs Technical Journal, vol. 1, no. 2, pp 41-59, Autumn 1996.

[4] G. Harikumar and Y. Bresler, "FIR Perfect Reconstruction from Multiple Convolutions: Minimum Deconvolver Orders", IEEE Trans. on Signal Processing, vol. 46, no. 1, pp. 215-218, 1998.

[5] Y. Inouye and K. Hirano, "Cumulant-Based Blind Identification of Linear Multi-Input-Multi-Output Systems Driven by Colored Inputs", IEEE Trans. on Signal Processing, vol. 45, pp. 1543-1552, 1997.

[6] Y. Li and K. Liu, "Adaptive Blind Source Separation and Equalization for Multiple-Input/Multiple-Output Systems", IEEE Trans. on Information Theory, vol. 44, no. 7, pp. 2864$2876,1998$.

[7] U. Lindgren and H. Broman, "Source Separation Using A Criterion Based on Second-Order Statistics", IEEE Trans, on Signal Processing, vol. 46, no. 7, pp. 1837-1850, 1998.

[8] H. Luo and R. Liu, "Blind Equalization for MIMO FIR Channels Based Only on Second Order Statistics by Use of Pre-Filters", in Proc. of SPAWC'99, pp. 106-109, Annapolis, MD, USA, 1999.

[9] J. Massey and M. Sain, "Inverses of linear sequential circuits", IEEE Trans. on Computers, pp. 330-337, 1968.

[10] A. Naguib, V. Tarokh, N. Seshadri, and A. R. Calderbank, "A Space-Time Coded Modem for High Data Rate Wireless Communication", IEEE Journal on Selected Areas in Communications, vol. 16, pp. 1459-1478, 1998.

[11] J. Tugnait, "Identification and Deconvolution of Multichannel Linear Non-Gaussian Processes Using Higher Order Statistics and Inverse Filter Criteria", IEEE Trans, on Signal Processing, vol. 45, pp. 658-672, 1997.

[12] J. Tugnait, "On Blind Identifiability of Multipath Channels Using Fractional Sampling and Second-Order Cyclostationary Statistics", IEEE Trans. on Information Theory, vol. 41, no. 1, pp. 308-311, 1995.

[13] J. Xavier, V. Barroso, and J. M. F. Moura, "Closed-form blind channel identification and source separation in SDMA systems through correlative coding", IEEE journal on Selected Areas in Communications, vol. 16, no. 8, pp. 1506-1517, 1998. 